\title{
Ein Mikrowellen-Mikrowellen-Doppelresonanz-Spektrograph mit gekreuzten Feldern
}

\author{
H. D. Rudolph, H. Dreizler * und U. Andresen * \\ Physikalisches Institut der Universität Freiburg \\ (Z. Naturforsch. 26 a, 233-239 [1971] ; eingegangen am 26. November 1970)
}

\begin{abstract}
Es wird ein Mikrowellen-Mikrowellen-Doppelresonanz-Spektrograph mit gekreuzten Feldern beschrieben. Dieser Spektrograph hat den Vorteil, daß vom experimentellen Aufbau her die Pumpund Signalfrequenz unabhängig voneinander sind. Die theoretische Linienform für gekreuzte Felder wird abgeleitet und den entsprechenden Messungen gegenübergestellt.
\end{abstract}

\section{Einleitung}

Die Doppelresonanzmethode ist heute in der Mikrowellenspektroskopie ein übliches Verfahren. Die theoretischen Grundlagen für das am häufigsten benutzte 3-Niveau-System stammen von $\mathrm{JAVAN}^{1}$ und Di Giacomo und BatTaglia ${ }^{2,3}$; die experimentellen Aufbauten von Cox et al. ${ }^{4}$, UnLAND et al. ${ }^{5}$ und Woods et al. ${ }^{6}$. Bei diesen Experimenten liegen sowohl Pump- als auch Signalfrequenz im Mikrowellengebiet.

Den gebräuchlichsten Aufbau zeigt Abb. 1 a. Vor dem als Absorptionszelle benutzten Hohlleiter wird

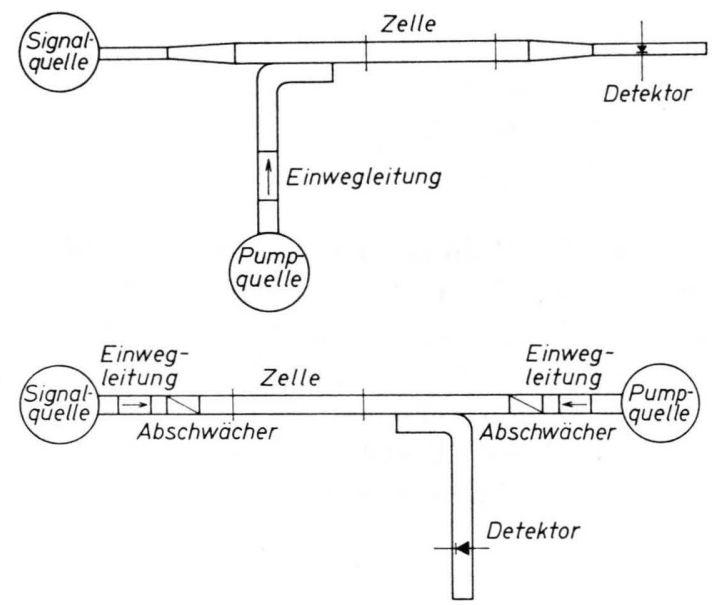

Abb. 1. Schematischer Aufbau eines MMDR-Spektrographen mit parallelen Feldern. a) Signalfrequenz höher als Pumpfrequenz; b) Signal- und Pumpfrequenz etwa gleich.

Sonderdruckanforderungen an Prof. Dr. H.-D. RuDolpH, Physikalisches Institut der Universität Freiburg, D-7800 Freiburg, Hermann-Herder-Str. 3.

* Jetzige Anschrift: Abt. Chemische Physik im Institut für Physikalische Chemie, D-2300 Kiel, Olshausenstr. 40/60.

1 A. Javan, Phys. Rev. 107, 1579 [1957].

2 A. Di Giacomo, Nuovo Cim. 14, 1082 [1952].

3 A. Battaglia, A. Di Giacomo u. S. Santuccio, Nuovo Cim. 43 B, 89 [1966]. die Pumpleistung über einen Richtkoppler eingekoppelt. Die Zelle muß so dimensioniert sein, daß sich sowohl Pump- als auch Signalfrequenz in ihr ausbreiten können. Ein Problem jedes Mikrowellen-Mikrowellen-Doppelresonanz-Spektrographen (MMDRSpektrograph) ist es, die Pumpstrahlung wirkungsvoll vom Detektor fernzuhalten. Liegt die Signalfrequenz ausreichend höher als die Pumpfrequenz, kann man die Existenz der Grenzfrequenz im Hohlleiter ausnutzen und den Empfänger in einem Hohlleiterstück unterbringen, in dem die Pumpfrequenz nicht ausbreitungsfähig ist.

Sind Pump- und Signalfrequenz annähernd gleich, kann man den Aufbau nach Abb. 1 b benutzen, wie er z. B. in ${ }^{4}$ beschrieben wird. Die Abtrennung der Pumpstrahlung ist hier merklich schlechter als im ersten Falle, da durch Reflexion an der Einwegleitung stets ein Teil der Pumpstrahlung zur Empfangsdiode gelangen kann.

Für den Fall, daß die Pumpfrequenz höher liegt als die Signalfrequenz, sind bisher nur sehr wenige Experimente bekannt geworden. Für diesen Aufbau wurden stets Filter benutzt. Die Schmalbandigkeit der Filter bedingt, daß ein Aufbau nur für einen engen Frequenzbereich verwendbar ist, was die Mes. sungen aufwendig und zeitraubend macht.

Ein vorteilhafterer Aufbau läßt sich durch geschicktes Ausnutzen der Grenzfrequenz erreichen, worüber wir in ${ }^{7}$ berichten. In einem Hohlleiter mit

4 A. P. Cox, W. G. Flynn u. E. B. Wilson, J. Chem. Phys. 42, 3094 [1965]

5 M. L. Unland, V. Weiss u. W. H. Flygare, J. Chem. Phys. 42, 2138 [1965].

${ }^{6}$ R. C. Woods III, A. M. Ronn u. E. B. Wilson, Rev. Sci. Instrum. 37, 927 [1966].

7 U. Andersen u. H. D. Rudolph, Z. Naturforsch. 26 a, 320 [1971]. 
dem Querschnitt $a \times b$ können sich nur Wellen mit der Frequenz

$$
v \geqq v_{\mathrm{g}}=c \sqrt{\left(\frac{m}{2 a}\right)^{2}+\left(\frac{n}{2 b}\right)^{2}}
$$

ausbreiten ${ }^{8}$. Für die praktisch stets benutzten $\mathrm{TE}_{10^{-}}$ Wellen $(m=1, n=0)$ ergibt sich daraus die Grenzfrequenz zu $v_{\mathrm{g}}=c / 2 a$. Die Dimension $a$ ist in diesem Falle die Seite des Hohlleiters, auf der die elek. trischen Feldlinien senkrecht stehen; bei den Normhohlleitern ist dieses üblicherweise die Breitseite. Die $b$-Dimension spielt für die Grenzfrequenz keine Rolle. Polarisiert man also die Pump- und Signalstrahlung senkrecht zueinander, so hat die eine Strahlung $\mathrm{TE}_{10^{-}}$und die andere $\mathrm{TE}_{01}$-Modus. Damit kann die Grenzfrequenz für die Pumpstrahlung ohne Rücksicht auf die Frequenz der Signalstrahlung gewählt werden.

Den schematischen Hohlleiterzug für den Fall, daß die Signalfrequenz höher als die Pumpfrequenz liegt, zeigt Abb. 2.

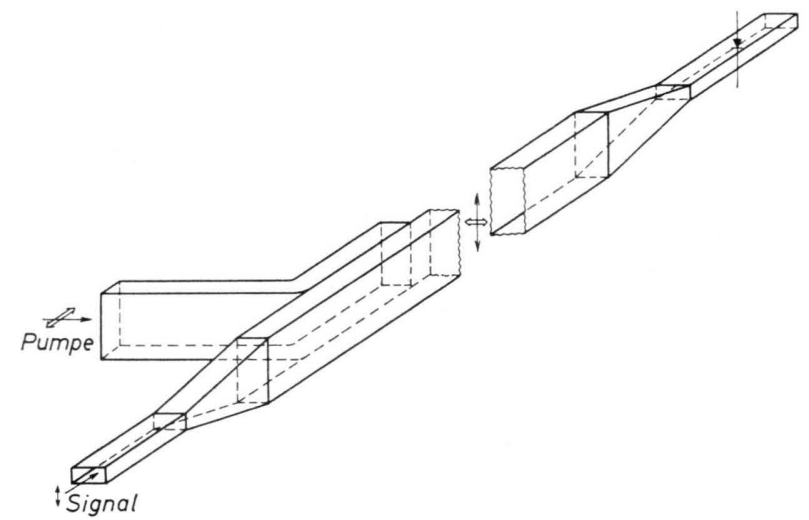

Abb. 2. Schematischer Hohlleiterzug für einen MMDR-Spektrographen mit gekreuzten Feldern.

Den Aufbau kann man - bis auf die Übergangsstücke - bequem mit Normhohlleitern bewältigen.

Benutzt man für die Zelle einen X-Band-Normhohlleiter $\left(a^{*} \times b^{*}=22,86 \times 10,16 \mathrm{~mm}^{2}\right)$ und für den Empfänger K-Band-Norm $(\bar{a} \times \bar{b}=10,67 \times 4,32$ $\mathrm{mm}^{2}$ ) (Abb. 2), so steht für die Pumpfrequenz der Bereich von ca. $6,5 \mathrm{GHz}\left(a^{*}\right)$ bis ca. $34 \mathrm{GHz}(\bar{b})$ und für die Signalfrequenz der Bereich oberhalb von ca. 14,5 GHz $(\bar{a})$ zur Verfügung.

8 D. Klages, Einführung in die Mikrowellenphysik, Steinkopff, Darmstadt 1965.

\section{Theorie}

Die theoretischen Arbeiten über Doppelresonanz (vgl. ${ }^{1-3}$ ) gehen von parallel polarisierten Pumpund Signalwellen aus. Es ist daher notwendig, entsprechende Überlegungen auch für den Fall der senkrecht zueinander polarisierten Wellen anzustellen. Dabei soll analog zu JAVAN ${ }^{1}$ vorgegangen werden.

Unter der Voraussetzung, daß für das Gas die Boltzmann-Verteilung gilt, und die Stöße statistisch erfolgen, zeigt Javan, daß das Gas beim Übergang von Niveau $a$ nach $b$ die Leistung

$P=(n(a)-n(b)) \frac{\hbar \omega_{0}}{\tau^{2}} \int_{-\infty}^{t}\left|c(a)\left(t-t_{0}\right)\right|^{2} e^{-\left(t-t_{0}\right) / \tau} \mathrm{d} t_{0}$

aus der Mikrowelle aufnimmt. Dabei ist $n(a)$ bzw. $n(b)$ die Besetzungszahldichte der Niveaus, $\tau$ die mittlere Zeit zwischen zwei Stößen, $\hbar \omega_{0}=E(b)-E(a)$ die Energiedifferenz zwischen den beiden Niveaus und $\left|c(a)\left(t-t_{0}\right)\right|^{2}$ die zeitabhängige Übergangswahrscheinlichkeit von $a$ nach $b$. Diese Formel gilt allgemein für einen Übergang; die entscheidende Größe ist also die Übergangswahrscheinlichkeit

$$
\left|c(a)\left(t-t_{0}\right)\right|^{2} \text {. }
$$

Die Wellenfunktion eines Moleküls, das sich zur Zeit $t_{0}=0$ in einem beliebigen stationären Anfangszustand $\psi(n, m)$ befand, wird unter dem Einfluß der Strahlung zur Zeit $t$

$$
\varphi=\sum_{n, m} c(n, m)(t) \psi(n, m) e^{-(i / \hbar) E(n) t},
$$

wobei $m$ die Richtungsquantenzahl bezüglich der raumfesten $Z$-Achse und $n$ Repräsentant der übrigen Quantenzahlen ist. Das Quadrat der zeitabhängigen Amplitude $c(n, m)(t)$ ist die Wahrscheinlichkeit, das Molekül zum Zeitpunkt $t$ im Zustand $\psi(n, m)$ zu finden. Durch Einsetzen in die zeitabhängige Schrödinger-Gleichung erhält man

$$
\begin{gathered}
\dot{c}\left(n^{\prime}, m^{\prime}\right)(t)=-\frac{i}{\hbar} \sum_{n, m} c(n, m)(t) e^{(i / \hbar)\left(E\left(n^{\prime}\right)-E(n)\right)^{t}} \\
\cdot\left\langle n^{\prime}, m^{\prime}|V(t)| n, m\right\rangle
\end{gathered}
$$

mit $V(t)=\mu\left(E_{0}{ }^{\prime} \sin \omega^{\prime} t+E_{0} \sin \omega t\right), \omega$ bzw. $\omega^{\prime}$ Frequenz der beiden eingestrahlten Wellen, $E_{0}$ bzw. $E_{0}{ }^{\prime}$ die Amplitude der Feldstärke.

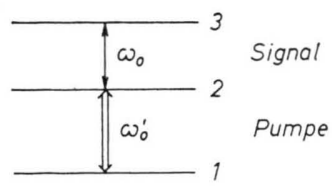

Abb. 3. 3-Niveau-Schema für Doppelresonanz. 
Da die Signal- und die Pumpfrequenz in der Nähe der jeweiligen Übergangsfrequenz liegen $\left(\omega^{\prime}\right.$ nahe $\omega_{0}{ }^{\prime}$ und $\omega$ nahe $\omega_{0}$ ), braucht man in (3) bei der Auswertung der Summe über $n$ nur die verbundenen Niveaus zu berücksichtigen. Für ein 3-Niveau-System (Abb. 3) ergibt sich dann $\left(E_{0} \sin \omega t\right.$ Signalstrahlung, $E_{0}{ }^{\prime} \sin \omega^{\prime} t$ Pumpstrahlung)

$$
\begin{gathered}
\bullet\left(1, m^{\prime}\right)(t)=-\frac{i}{\hbar} \sum_{m} c(2, m)(t) e^{-i \omega_{0}{ }^{\prime} t} \\
\cdot\left\langle 1, m^{\prime}|\mu| 2, m\right\rangle E_{0}{ }^{\prime} \sin \omega^{\prime} t, \\
\qquad\left(2, m^{\prime}\right)(t)=-\frac{i}{\hbar} \sum_{m}\left\{c(1, m)(t) e^{+i \omega_{0}{ }^{\prime} t}\right. \\
\cdot\left\langle 2, m^{\prime}|\mu| 1, m\right\rangle E_{0}{ }^{\prime} \sin \omega^{\prime} t \quad(4) \\
\left.+c(3, m)(t) e^{-i \omega_{0} t}\left\langle 2, m^{\prime}|\mu| 3, m\right\rangle E_{0} \sin \omega t\right\}, \\
\cdot\left(3, m^{\prime}\right)(t)=-\frac{i}{\hbar} \sum_{m} c(2, m)(t) e^{+i \omega_{0} t} \\
\left.\cdot \cdot 3, m^{\prime}|\mu| 2, m\right\rangle E_{0} \sin \omega t .
\end{gathered}
$$

Rechnet man $\sin \omega^{\prime} t$ in die entsprechende Exponentialfunktion um, und läßt die Glieder mit $e^{ \pm i\left(\omega^{\prime}+\omega_{0}^{\prime}\right) t}$ als schnellveränderliche Störung fort, so erhält man endgültig

$$
\begin{aligned}
\dot{c}\left(1, m^{\prime}\right)(t) & =-\sum_{m} c(2, m)(t) y\left(m^{\prime}, m\right) e^{+i\left(\omega^{\prime}-\omega_{0}^{\prime}\right) t}, \\
\dot{c}\left(2, m^{\prime}\right)(t) & =+\sum_{m}\left\{c(1, m)(t) y^{*}\left(m, m^{\prime}\right) e^{-i\left(\omega^{\prime}-\omega_{0}^{\prime}\right) t}\right. \\
& \left.-c(3, m)(t) x\left(m^{\prime}, m\right) e^{+i\left(\omega-\omega_{0}\right) t}\right\},
\end{aligned}
$$

$\stackrel{\bullet}{c}\left(3, m^{\prime}\right)(t)=+\sum_{m} c(2, m)(t) x^{*}\left(m, m^{\prime}\right) e^{-i\left(\omega-\omega_{0}\right) t}$.

mit $2 \hbar y\left(m^{\prime}, m\right)=\left\langle 1, m^{\prime}|\mu| 2, m\right\rangle E_{0}{ }^{\prime}$,

$$
2 \hbar x\left(m^{\prime}, m\right)=\left\langle 2, m^{\prime}|\mu| 3, m\right\rangle E_{0} .
$$

In Gl. (5) ist die Hermitizität der Dipolmatrix ausgenutzt worden.

Das System gekoppelter Diff.-Gln. (5) gestattet es prinzipiell, bei Kenntnis der Dipolmatrixelemente die Übergangswahrscheinlichkeiten $|c(n, m)(t)|^{2} \mathrm{zu}$ berechnen. Eine geschlossene Lösung ist für belie- bige $x\left(m^{\prime}, m\right)$ im allgemeinen nicht möglich. Ist jedoch $|y| \gg|x|$, was sich stets durch entsprechend große Pumpleistungen erreichen läßt, kann man in den Gleichungen, in denen Ausdrücke in $x$ und in $y$ vorkommen, die Glieder mit $x$ vernachlässigen. Dadurch reduziert sich die Kopplung und geschlossene Lösungen sind möglich.

Die für die einleitend besprochene Konzeption wesentliche Doppelresonanz mit gekreuzten Feldern wurde am Propylenoxyd ${ }^{9}$ nachgewiesen. Das benutzte System sieht wie folgt aus:

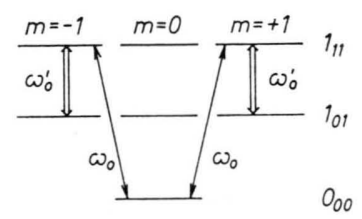

Abb. 4. 3-Niveau-System des Propylenoxyds zum Nachweis der Doppelresonanz mit gekreuzten Feldern; Pumpfeld in $Z$-Richtung, Signalfeld in $Y$-Richtung. $0_{00}-1_{11}: \mu_{\mathrm{b}}$-Übergang; $1_{01}-1_{11}: \mu_{\mathrm{c}}$-Ưbergang; $\uparrow$ Signalübergang, $\uparrow$ Pumpübergang.

Die Z-Richtung wurde dabei ohne Einschränkung der Allgemeinheit parallel zum elektrischen Feldvektor der Pumpe, die $Y$-Richtung parallel zum elektrischen Feldvektor des Signals gewählt. Eine andere Festsetzung würde zu denselben Endformeln führen. Die Auswahlregeln für die hier angenommene Orientierung lauten

$$
\begin{array}{ll}
m=m^{\prime} \pm 1 & \text { für die } Y \text {-Komponente } \mu_{Y}, \\
m=m^{\prime} & \text { für die } Z \text {-Komponente } \mu_{Z} \\
& \text { mit } 0 \leftarrow \rightarrow 0 \text { für } \Delta J=0 .
\end{array}
$$

Für diesen Fall erhält man von (3) ausgehend folgende fünf gekoppelten Diff.-Gln.:

$$
\begin{gathered}
\dot{c}\left(0_{00}, 0\right)(t)=-\left\{c\left(1_{11},+1\right)(t) x(0,+1)\right. \\
\left.\quad+c\left(1_{11},-1\right) x(0,-1)\right\} e^{+i\left(\omega-\omega_{0}\right) t}, \\
\dot{c}\left(1_{01}, \pm 1\right)(t)= \\
\quad-c\left(1_{11}, \pm 1\right)(t) y( \pm 1, \pm 1) e^{+i\left(\omega^{\prime}-\omega_{0}^{\prime}\right) t}
\end{gathered}
$$

$$
\stackrel{\bullet}{c}\left(1_{11}, \pm 1\right)(t)=+c\left(1_{01}, \pm 1\right)(t) y^{*}( \pm 1, \pm 1) \cdot e^{-i\left(\omega^{\prime}-\omega_{0}^{\prime}\right) t}+c\left(0_{00}, 0\right)(t) x^{*}(0, \pm 1) e^{-i\left(\omega-\omega_{0}\right) t} .
$$

Dabei gilt entweder das obere oder das untere Vorzeichen. Ausgenutzt wurde hier, daß eine Reihe von in (5) formal vorhandenen Matrixelementen verschwindet.

Bei einem 3-Niveau-System sind zwei Übergänge möglich, die zur Wechselwirkung mit der Signalstrahlung beitragen: Einmal der normale, direkte
Übergang - in unserem Fall zwischen $1_{11}$ und $0_{00}$; zum anderen besteht die Möglichkeit, daß ein Molekül in einem Zweiquantenprozeß zuerst durch Wechselwirkung mit der Pumpstrahlung von $l_{01}$ nach

9 J. D. Swalen u. D. R. Herschbach, J. Chem. Phys. 27, 100 [1957]. 
$\mathrm{l}_{11}$ und dann durch Wechselwirkung mit der Signalstrahlung in den Zustand $0_{00}$ übergeht.

Für Moleküle, die sich zur Zeit $t_{0}=0$ im Zustand $1_{01}$ befinden, gilt:

$$
\begin{aligned}
& c\left(1_{01}, \pm 1\right)\left(t_{0}=0\right)=1, \\
& c\left(1_{11}, \pm 1\right)\left(t_{0}=0\right)=c\left(0_{00}, 0\right)\left(t_{0}=0\right)=0 .
\end{aligned}
$$

$\left|c\left(0_{00}, 0\right)(t)\right|^{2}$ ist dann die Wahrscheinlichkeit, das Molekül zur Zeit $t$ im Zustand $0_{00}$ zu finden.

Unter der oben diskutierten Vernachlässigung für $|x| \ll|y|$ erhält man aus (7) und den Anfangsbedingungen (8) (Index $Z$ für Zweiquantenübergang) :

$$
\begin{aligned}
&\left|c\left(0_{00}, 0\right)(t)\right|_{Z}^{2}=\frac{1}{\gamma^{2}} \mid x(0,+1) y^{*}(+1,+1)+\left.x(0,-1) y^{*}(-1,-1)\right|^{2} \\
& \cdot\left\{\begin{array}{c}
\frac{\sin ^{2} \frac{1}{2}(\gamma-\alpha) t}{(\gamma-\alpha)^{2}}+\frac{\sin ^{2} \frac{1}{2}(\gamma+\alpha) t}{(\gamma+\alpha)^{2}} \\
+\frac{\cos ^{2} \gamma t-\frac{1}{2}[\cos (\gamma-\alpha) t+\cos (\gamma+\alpha) t]}{\gamma^{2}-\alpha^{2}}
\end{array}\right\}
\end{aligned}
$$

mit

$$
\begin{aligned}
& \gamma=\frac{1}{2}\left[\left(\omega^{\prime}-\omega_{0}{ }^{\prime}\right)+4|y(1,1)|^{2}\right]^{1 / 2} ; \\
& \alpha=-\frac{1}{2}\left(\omega^{\prime}-\omega_{0}{ }^{\prime}\right)+\left(\omega-\omega_{0}\right) .
\end{aligned}
$$

Für Moleküle, die sich zur Zeit $t_{0}=0 \mathrm{im}$ Zustand $\mathrm{l}_{11}$ befinden, gilt:

$$
\begin{aligned}
& c\left(1_{11}, \pm 1\right)\left(t_{0}=0\right)=1, \\
& c\left(1_{01}, \pm 1\right)\left(t_{0}=0\right)=c\left(0_{00}, 0\right)\left(t_{0}=0\right)=0 .
\end{aligned}
$$

Die Wahrscheinlichkeit, daß sich ein solches Molekül zur Zeit $t$ im Zustand $0_{00}$ befindet, d. h. die Übergangswahrscheinlichkeit für den Einquantenprozeß, ist:

$$
\begin{aligned}
& \left|c\left(0_{00}, 0\right)(t)\right|_{E}^{2}=|x(0,+1)+x(0,-1)|^{2} \\
& \cdot\left\{(1-a)^{2} \frac{\sin ^{2} \frac{1}{2}(\gamma-\alpha) t}{(\gamma-\alpha)^{2}}+(1+a)^{2} \frac{\sin ^{2} \frac{1}{2}(\gamma+\alpha) t}{(\gamma+\alpha)^{2}}\right. \\
& \left.\quad-\left(1-a^{2}\right) \frac{\cos ^{2} \gamma t-\frac{1}{2}[\cos (\gamma-\alpha) t+\cos (\gamma+\alpha) t]}{\gamma^{2}-\alpha^{2}}\right\}(11)
\end{aligned}
$$

mit $a=\left(\omega^{\prime}-\omega_{0}^{\prime}\right) / 2 \gamma$.

Da die Übergangswahrscheinlichkeit für Emission und Absorption gleich ist, kann man mit (9) und (11) aus (1) die absorbierte Leistung für den Zweiquanten- und für den Einquantenprozeß angeben:

$$
\begin{aligned}
& P_{Z}=\left(n\left(0_{00}\right)-n\left(1_{01}\right)\right) \hbar \omega_{0} \tau\left|x(0,+1) y^{*}(+1,+1)+x(0,-1) y^{*}(-1,-1)\right|^{2} \\
& \quad \cdot \frac{1}{\gamma^{2}}\left\{\frac{1}{2\left[1+(\gamma+\alpha)^{2} \tau^{2}\right]}+\frac{1}{2\left[1+(\gamma-\alpha)^{2} \tau^{2}\right]}+\frac{\left(\gamma^{2}-\alpha^{2}\right) \tau^{2}\left(1+2 \gamma^{2} \tau^{2}\right)-1}{\left(1+4 \gamma^{2} \tau^{2}\right)\left[1+(\gamma+\alpha)^{2} \tau^{2}\right]\left[1+(\gamma-\alpha)^{2} \tau^{2}\right]}\right\} ; \\
& \quad P_{\mathrm{E}}=\left(n\left(0_{00}\right)-n\left(1_{11}\right)\right) \hbar \omega_{0} \tau|x(0,+1)+x(0,-1)|^{2} \\
& \quad \cdot\left\{\frac{(1+a)^{2}}{2\left[1+(\gamma+\alpha)^{2} \tau^{2}\right]}+\frac{(1-a)^{2}}{2\left[1+(\gamma-\alpha)^{2} \tau^{2}\right]}-\left(1-a^{2}\right) \frac{\left(\gamma^{2}-\alpha^{2}\right) \tau^{2}\left(1+2 \gamma^{2} \tau^{2}\right)-1}{\left(1+4 \gamma^{2} \tau^{2}\right)\left[1+(\gamma+\alpha)^{2} \tau^{2}\right]\left[1+(\gamma-\alpha)^{2} \tau^{2}\right]}\right\} .
\end{aligned}
$$

Die Form der Doppelresonanz ergibt sich aus der Überlagerung von (12) und (13). Es treten zwei Absorptionsmaxima auf, und zwar für (12) und (13) jeweils bei:

d. h.

$$
\gamma \pm \alpha=0
$$

$$
\begin{array}{r}
\omega=\omega_{0}+\frac{1}{2}\left(\omega^{\prime}-\omega_{0}{ }^{\prime}\right) \mp \frac{1}{2}\left[\left(\omega^{\prime}-\omega_{0}{ }^{\prime}\right)^{2}\right. \\
\left.+4|y(1,1)|^{2}\right]^{1 / 2}
\end{array}
$$

man erhält also eine Dublettstruktur. Ist $|y|^{2} \tau^{2} \gg 1$, d. h. die Pumpleistung genügend hoch, was schon früher gefordert wurde, kann man den letzten Term von (12) und (13) vernachlässigen, was im folgenden getan wird. Dasselbe gilt bei niedrigem Druck, d. h. $\tau$ groß.

Die Aufspaltung in das Dublett erfolgt symmetrisch zu $\omega_{0}+\frac{1}{2}\left(\omega^{\prime}-\omega_{0}^{\prime}\right)$. Ist die Pumpe in Resonanz $\left(\omega^{\prime}=\omega_{0}{ }^{\prime}\right)$, so ist das Dublett symmetrisch zu $\omega_{0}$, der Lage der normalen „ungepumpten“ Absorptionslinie, und die Aufspaltung hängt nur von der Leistung der Pumpe ab. Verschiebt man die Pump- frequenz, so wird auch die Frequenzmitte des Dubletts verschoben, und zwar um $\frac{1}{2}\left|\omega^{\prime}-\omega_{0}^{\prime}\right|$. Die Richtung der Verschiebung hängt von der Anordnung der Energieniveaus ab und stimmt in unserem Fall mit der Verschiebungsrichtung der Pumpe überein. Gleichzeitig verändern sich auch die Größe der Aufspaltung und die Intensität der beiden Komponenten. Für das Intensitätsverhältnis beider Komponenten gilt für den Einquantenprozeß

$$
\frac{I_{\mathrm{E}+}}{I_{\mathrm{E}-}}=\frac{(1+a)^{2}}{(1-a)^{2}}=\frac{\gamma^{2}+\left(\omega^{\prime}-\omega_{0}{ }^{\prime}\right)+\frac{1}{4}\left(\omega^{\prime}-\omega_{0}{ }^{\prime}\right)^{2}}{\gamma^{2}-\left(\omega^{\prime}-\omega_{0}{ }^{\prime}\right)+\frac{1}{4}\left(\omega^{\prime}-\omega_{0}\right)^{2}} .
$$

Für den Doppelquantenprozeß bleibt das Verhältnis stets gleich, jedoch ist die Intensität proportional zu $\left[\frac{1}{4}\left(\omega^{\prime}-\omega_{0}{ }^{\prime}\right)+|y|^{2}\right]^{-1}$. Verschiebt man die Pumpfrequenz zu höheren Frequenzen, so wandert die frequenzniedrige Komponente zur Resonanzstelle $\omega_{0}$ und wird dabei intensiver, während die andere Komponente zu höheren Frequenzen verschoben wird und dabei rasch an Intensität abnimmt (Abb. 5). Diese Komponente verschiebt sich bezüglich $\omega_{0}$ 

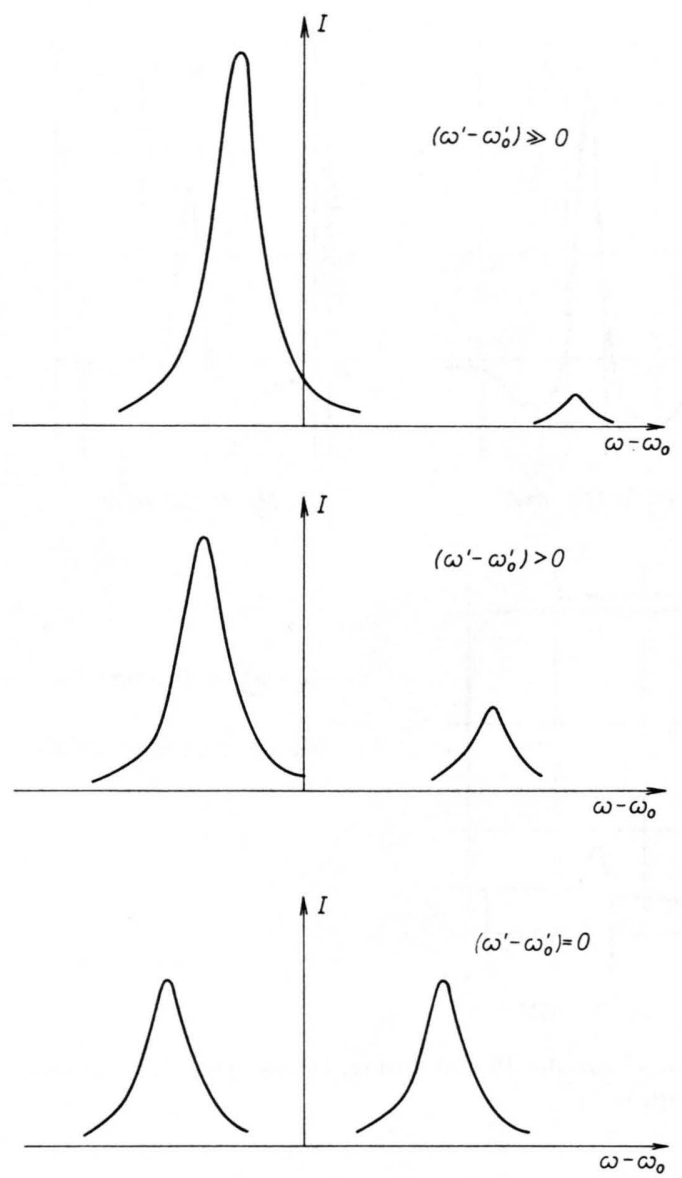

Abb. 5. Abhängigkeit des Signalübergangs von der Ablage $\left(\omega^{\prime}-\omega_{0}{ }^{\prime}\right)$ der Pumpfrequenz von der Resonanzstelle $\omega_{0}{ }^{\prime}$; vgl. Gln. (12) und (13).

sehr stark; sie wird „creeper“ genannt. Verschiebt man die Pumpfrequenz zu niedrigeren Frequenzen, vertauschen die beiden Linien ihre Rollen. Wie man es erwartet, trägt der Doppelquantenprozeß umso weniger zur Linienform bei, je weiter die Pumpe aus der Resonanz entfernt wird.

Alle diese Effekte sind qualitativ dieselben, wie sie auch für Doppelresonanz bei parallelen Feldern gefunden wurden.

Die Abb. 6 und 7 zeigen die Messungen für die oben diskutierten Fälle. Die Linienform ist durch die Überlagerung zweier Effekte entstanden (siehe unten) :

a) Absorption mit starker Pumpe,

b) Absorption ohne Pumpe.

Die normale Absorptionslinie wird dabei nach oben geschrieben, die Doppelresonanzlinie nach unten.

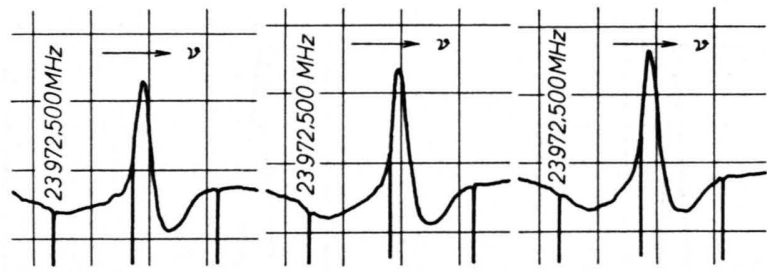

$$
\Delta v_{p}=-2000 \mathrm{kHz} \quad \Delta v_{p}=-1500 \mathrm{kHz} \quad \Delta v_{p}=-1000 \mathrm{kHz}
$$

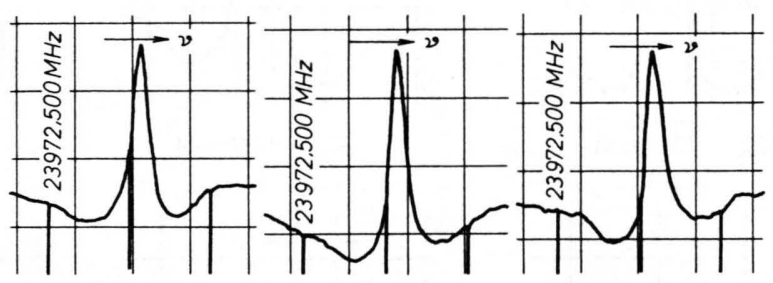

$\Delta v_{p}=-500 \mathrm{kHz} \quad \Delta v_{p}=0 \mathrm{kHz} \quad \Delta v_{p}=500 \mathrm{kHz}$

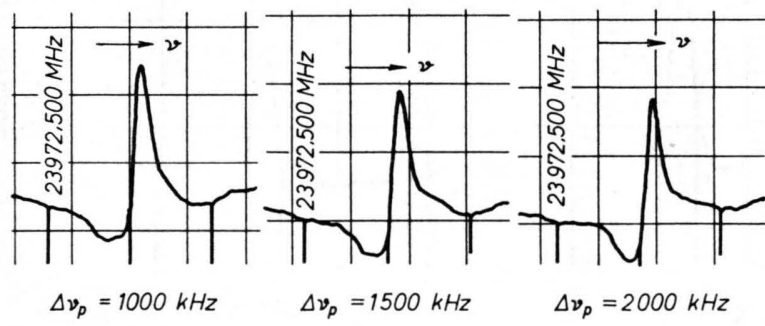

$\Delta v_{p}=v_{p}-v_{p}^{0}$

Pumpleistung: $100 \mathrm{~mW}$

Markenabstand: $2500 \mathrm{kHz}$

Abb. 6. Beobachteter Signalübergang $\left(0_{00}-1_{11}\right)$ von Propylenoxyd, Pumpe $\left(1_{01}-1_{11}\right)$ moduliert und schrittweise durch die Resonanz durchgestimmt.

Bei der Auswahl von Doppelresonanzsystemen ist eine genaue Beachtung der Auswahlregeln wichtig. Zum Beispiel kann man bei parallel polarisierten Wellen wegen der anderen Auswahlregeln an dem beschriebenen Niveau-System keinen Doppelresonanzeffekt nachweisen, wie Abb. 8 zeigt.

\section{Experimentelles}

Da die Pumpfrequenz mit $11341,6 \mathrm{MHz}\left(1_{10}-1_{11}\right)$ im X-Band und die Signalfrequenz mit $23975,2 \mathrm{MHz}$ $\left(0_{00}-1_{11}\right)$ im K-Band liegt, wurde der Aufbau nach Abb. 2 verwirklicht. Das Blockschaltbild des Gesamtaufbaus zeigt Abb. 9.

Als Zelle wurde ein X-Band-Hohlleiter von $5 \mathrm{~m}$ Länge benutzt. Um die bekannten Vorteile molekularer Modulation und des phasenempfindlichen Empfangs ausnutzen zu können, wurde die Pumpe ein/aus- moduliert ${ }^{6}$. Wesentlich ist, daß dann nur die Doppelresonanzlinie registriert wird. Alle anderen Absorp- 


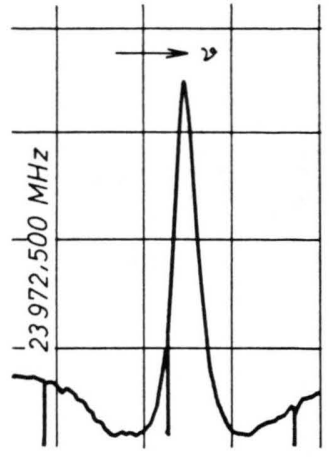

$P_{p}=170 \mathrm{~mW}$

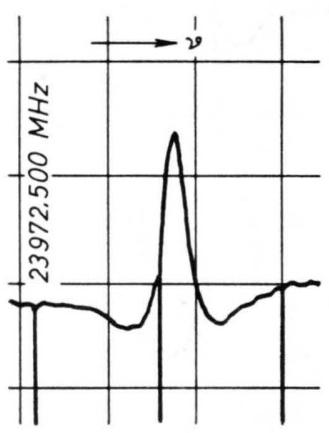

$P_{p}=50 \mathrm{~mW}$

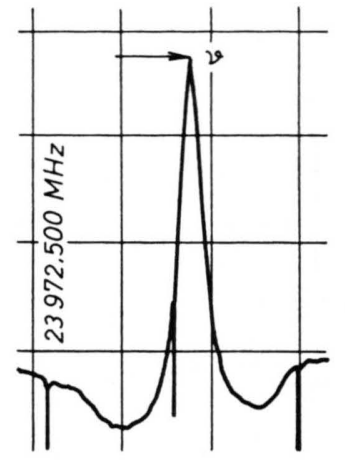

$P_{p}=140 \mathrm{~mW}$

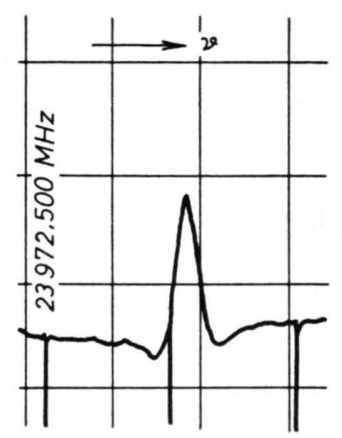

$P_{p}=20 \mathrm{~mW}$

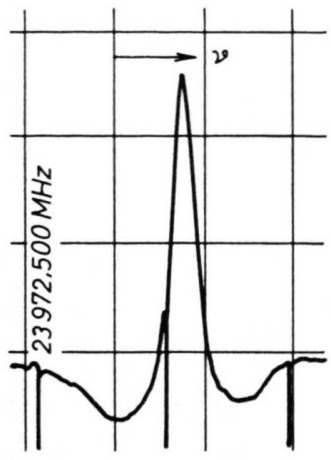

$P_{p}=110 \mathrm{~mW}$

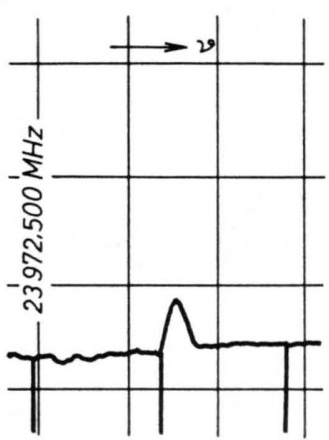

$P_{p}=2 \mathrm{~mW}$

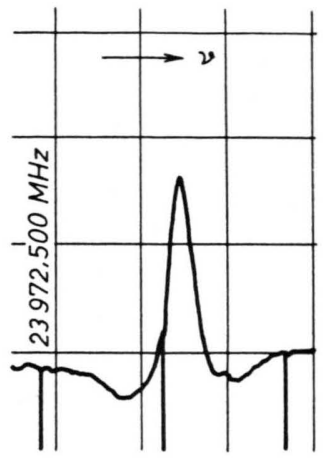

$P_{p}=80 \mathrm{~mW}$

$v_{p}-v_{p}^{0}=0 \mathrm{kHz}$

Markenabstand: $2500 \mathrm{kHz}$

Abb. 7. Abhängigkeit des Signalübergangs $\left(0_{00}-l_{11}\right)$ von Propylenoxyd von der Pumpleistung, Pumpe $\left(1_{01}-l_{11}\right)$ konstant in Resonanz $\left(\omega^{\prime}=\omega_{0}^{\prime}\right)$.

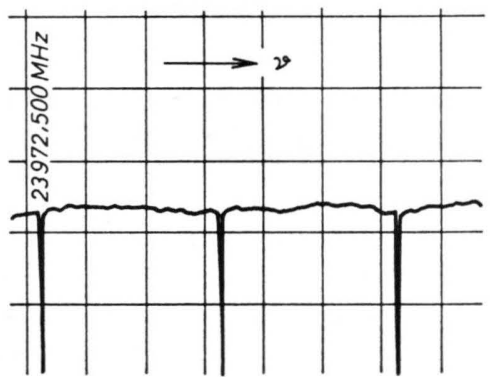

Pumpleistung: $160 \mathrm{~mW}$

Markenabstand: $2500 \mathrm{kHz}$

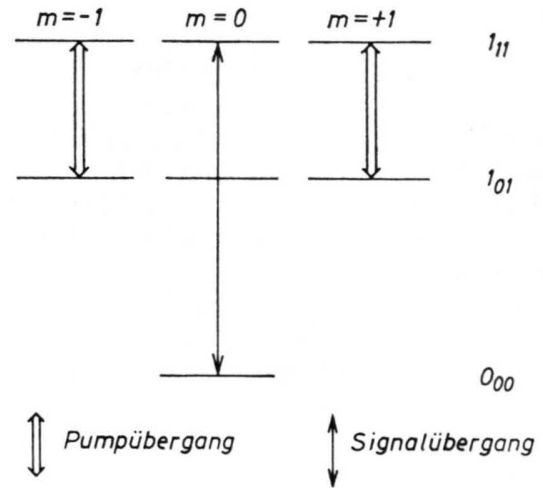

$\leftarrow$ Abb. 8. Bei parallelen Feldern von Signal- und Pumpstrahlung erhält man keine Doppelresonanz, da kein gemeinsames Niveau existiert (für $\Delta J=0$ ist $m: 0-0$ nicht erlaubt).

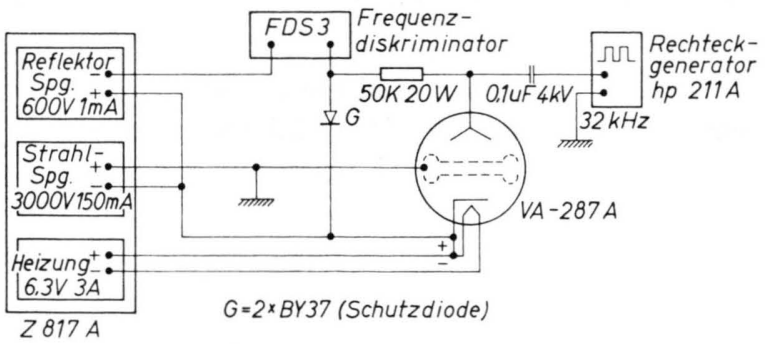

Abb. 10. Blockschaltbild der Pumpquelle mit Stabilisation und Modulation. 


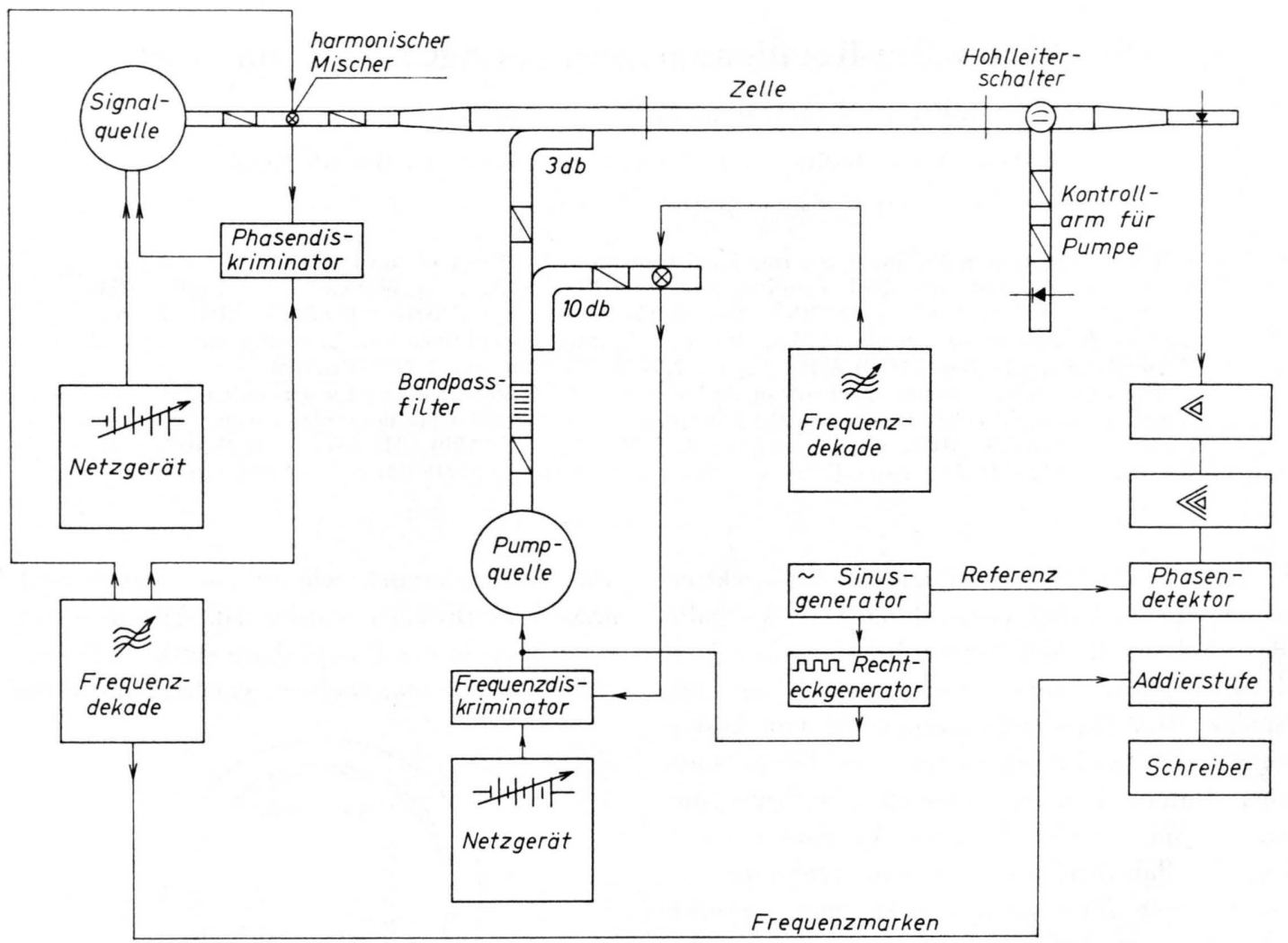

Abb. 9. Blockschaltbild des Gesamtaufbaues.

tionslinien, auch eine eventuell den Doppelresonanzeffekt überdeckende Störlinie, sind nicht moduliert und ergeben kein Signal. Dieses Verfahren bringt mit sich, daß während der Aus-Phase der Pumpe die normale Absorptionslinie erscheint; es wird also stets die Superposition von normaler Linie und Doppelresonanzlinie gemessen.

Als Pumpquelle wurde ein Klystron VA $287 \mathrm{~A} *$ * mit $1 \mathrm{~W}$ Ausgangsleistung benutzt. Zur Ein/Aus-Modulation wird zusätzlich auf den Reflektor eine Rechteckspannung von ca. $50 \mathrm{~V}$ gegeben. Dadurch wird erreicht, daß das Klystron periodisch aus dem Schwingungsmode gezogen wird, was eine Modulation von $100 \%$ garantiert. Gleichzeitig wird die Pumpquelle noch mittels eines Frequenzstandards stabilisiert. Da das Anschwingen des Klystrons ohne feste Phasenbeziehung zur vorhergehenden Schwingungsperiode geschieht, kann

**Fa. V a ria n. dazu nur ein Frequenz- und kein Phasendiskriminator benutzt werden. Die vollständige Schaltung der Pumpquelle zeigt Abb. 10.

Die Pumpleistung wird über einen $3 \mathrm{db}$ Mehrlochrichtkoppler in die Zelle eingekoppelt. Dieses bedeutet, daß nur maximal 50\% der Pumpleistung in die Zelle gelangen, der Rest wird in dem eingebauten Wellensumpf vernichtet.

Der restliche Aufbau entspricht bis auf die Übergangsstücke einem üblichen Stark-Effekt-Spektrographen. Eine ausführliche Diskussion, insbesondere auch der experimentellen Probleme findet man in ${ }^{\mathbf{1 0}}$.

Wir danken der Deutschen Forschungsgemeinschaft und dem Fonds der Chemischen Industrie für Sachmittel.

10 U. Andresen, Diplomarbeit, Freiburg 1969. 